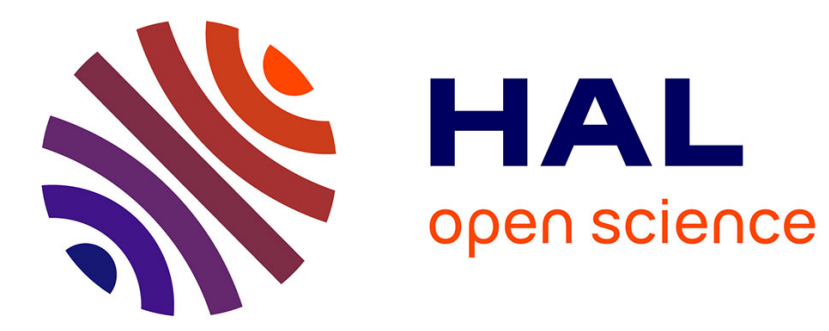

\title{
Les phénomènes de substitution fourrages-concentrés chez la vache laitière
}

Philippe Faverdin, J.P. Dulphy, J.B. Coulon, R. Vérité, J.P. Garel, J. Rouel, B. Marquis

\section{- To cite this version:}

Philippe Faverdin, J.P. Dulphy, J.B. Coulon, R. Vérité, J.P. Garel, et al.. Les phénomènes de substitution fourrages-concentrés chez la vache laitière. Productions Animales, 1992, 5 (2), pp.127-135. hal-00895969

\section{HAL Id: hal-00895969 \\ https://hal.science/hal-00895969}

Submitted on 1 Jan 1992

HAL is a multi-disciplinary open access archive for the deposit and dissemination of scientific research documents, whether they are published or not. The documents may come from teaching and research institutions in France or abroad, or from public or private research centers.
L'archive ouverte pluridisciplinaire HAL, est destinée au dépôt et à la diffusion de documents scientifiques de niveau recherche, publiés ou non, émanant des établissements d'enseignement et de recherche français ou étrangers, des laboratoires publics ou privés. 
INRA Prod. Anim., 1992, 5 (2), 127 - 135
P. FAVERDIN, J.P. DULPHY *,

J.B. COULON **, R. VERITÉ,

J.P. GAREL ***, J. ROUEL ****, B. MARQUIS

INRA Station de Recherches sur la vache laitière 35590 Saint-Gilles

* INRA Station de Recherches sur la Nutrition des Herbivores

63122 Saint-Genès Champanelle

** INRA Laboratoire Adaption des

Herbivores aux milieux

63122 Saint-Genès Champanelle

*** INRA Domaine de Marcenat

15190 Marcenat

**** INRA Domaine d'Orcival

63210 Rochefort-Montagne
Les phénomènes de substitution fourragesconcentrés chez la vache laitière

\section{L'intérêt économique et technique d'un apport d'aliment concentré pour améliorer la valeur énergétique d'une ration de vache laitière dépend de la modification de consommation de fourrage qui s'ensuit. Ce phénomène de substitution du fourrage par de l'aliment concentré est donc important à connaître et à prévoir afin d'optimiser le rationnement des vaches laitières et de mieux prédire les quantités ingérées.}

Parmi les facteurs susceptibles de modifier la consommation de matière sèche par des ruminants, la quantité d'aliments concentrés offerte est sans doute l'un des plus importants. Très généralement, dans le cas de ration pour vaches laitières, l'accroissement des apports d'aliments concentrés s'accompagne d'une augmentation de la quantité de matière sèche totale ingérée mais d'une diminution des quantités de fourrage ingérées. Ce phénomène, appelé substitution fourrageconcentré, est estimé par le calcul d'un taux de substitution (TS) égal à la variation de

\section{Résumé}

Les principaux facteurs de variations des taux de substitution (TS) entre fourrage et aliments concentrés que constituent le niveau d'apport du concentré, la nature du fourrage et la nature de l'aliment concentré ont été étudiés au cours de 8 essais réalisés en 3 lieux différents. Dans chaque essai, 3 niveaux de d'apport de concentrés (bas, moyen et haut) ont été comparés selon un schéma carré latin 3x3, répété 3 ou 4 fois, avec des vaches pie-noires en milieu de lactation et au cours de périodes de 1 mois. Les 8 essais correspondaient à 8 régimes différant par la nature du fourrage (ensilage de maïs, ensilage d'herbe ou foin) et la nature du concentré (riche en amidon, riche en parois très digestibles, ou riche en parois moyennement digestibles). Quels que soient les fourrages et les concentrés, le taux de substitution augmente systématiquement avec le niveau d'apport de concentré : il passe, en moyenne, de 0,47 entre les niveaux bas et moyen à 0,67 entre les niveaux moyens et haut. Pour un fourrage donné, le taux de substitution est plus élevé lorsque la concentration énergétique du concentré est plus forte, mais cet écart n'existe qu'entre les niveaux bas et moyen d'apport. Les taux de substitution moyens observés sur ensilage de maïs $(0,70)$ sont supérieurs à ceux observés sur ensilage d'herbe $(0,53)$ ainsi que sur foin $(0,44)$. Ces essais montrent, à travers les 3 facteurs de variations étudiés, que les taux de substitution observés en milieu de lactation sont d'autant plus importants que le bilan énergétique des animaux est plus élevé. consommation de fourrage distribué à volonté rapportée à l'augmentation d'ingestion d'aliments concentrés.

Plusieurs facteurs de variations du TS sont déjà connus. L'ingestibilité des fourrages semble être l'un des plus importants (Montgomery et al 1971, Dulphy 1978, Thomas 1987). Des relations ont ainsi pu être établies entre les valeurs de TS et l'encombrement (fonction inverse de l'ingestibilité) des fourrages dans le système des unités d'encombrement (UE) (INRA 1979, Jarrige et al 1986). L'influence de la nature du fourrage dans les phénomènes de substitution n'est cependant probablement pas limitée à la seule caractéristique d'ingestibilité.

L'effet de la proportion d'aliments concentrés dans la ration dans les phénomènes de substitution a bien été démontré chez les moutons et les bovins en croissance ou à l'engrais (INRA 1979, Berge et Dulphy 1985, Jarrige et al 1986). Chez les vaches laitières, de nombreux auteurs ont observé un accroissement du TS lorsque l'apport d'aliments concentrés augmentait (Lamb et al 1973, Vérité et Journet 1973, Ostergaard 1979, Vinet et al 1980, Laird et al 1981). Cependant dans d'autres études le TS n'a pas varié avec la quantité d'aliments concentrés (Kristensen 1983, Gordon 1984), ou a varié de façon contradictoire (Steen et Gordon $1980 \mathrm{a}$ b, Bertilsson et Burstedt 1983, Butler et al 1983).

Enfin l'effet de la nature de l'aliment concentré a jusqu'ici été peu étudié. Les essais 
de Castle (1981), puis de Dulphy et al (1987) n'ont pas montré de différences nettes entre des concentrés riches en amidon (céréales) ou en parois végétales très digestibles (pulpes de betteraves déshydratées), mais ce résultat méritait d'être confirmé.

Une série d'essais a donc été réalisée en 1986 et 1987, afin d'estimer les variations de TS fonction du niveau d'apport d'aliment concentré, de la nature du fourrage (ensilage de maïs, ensilage d'herbe ou foin) et du type d'aliments concentrés utilisé (riche en amidon, en parois hautement digestibles ou en parois moyennement digestibles). Elle a servi de base à l'élaboration du nouveau système des UE (Dulphy et al 1987, Faverdin et al 1987). Les résultats de ces essais et leurs conditions d'obtention ont été présentés et discutés de façon détaillée par Faverdin et al (1991), auquel on pourra se reporter.

\section{Conditions expérimentales}

Huit essais construits suivant un schéma en carré latin $3 \times 3$ avec 3 répétitions en moyenne ont été réalisés dans trois sites d'expérimentation différents (Rennes, Orcival et Marcenat) de façon à pouvoir utiliser plusieurs types de fourrages avec des animaux habitués à recevoir ces rations. Dans chaque essai, 3 quantités d'aliments concentrés (Bas $=\mathrm{B}$, Moyen $=\mathrm{M}$, Haut $=\mathrm{H}$ ) ont été distribuées pendant des périodes de 4 semaines. Dans tous les essais, le niveau haut correspondait au niveau moyen d'apport d'aliment concentré +2 UFL d'aliment concentré expérimental et le niveau bas au niveau moyen - 2 UFL.

Trois fourrages (ensilage de maïs, ensilage d'herbe ou foin) et 3 types d'aliments concentrés ont été comparés (riche en amidon, en parois très digestibles ou en parois peu digestibles). Il n'a pas été possible de croiser totalement ces 2 facteurs, et le régime à base de foin n'a été utilisé qu'avec le concentré de type amidon (tableau 1). Les niveaux moyens d'aliments concentrés ont été calculés individuellement en fonction de la nature des aliments et du potentiel de production des animaux afin de rendre les TS calculés comparables entre eux. Les valeurs énergétiques des 3 aliments concentrés utilisés étant différentes, les quantités d'aliments concentrés offertes ont donc été fonction de leur nature.

En début d'essai, les animaux, de type Pie Noir, étaient en moyenne entre leur deuxième et leur quatrième mois de lactation avec un niveau de production moyen variant de 23 à $34 \mathrm{~kg} / \mathrm{j}$ suivant les essais. Chaque bloc d'un carré latin a été constitué sur la base du niveau de production, de la date de vêlage, du numéro de lactation, du poids vif et des quantités ingérées. Les caractéristiques moyennes des animaux utilisés dans chaque essai sont précisées au tableau 1.

Les animaux étaient alimentés individuellement en stabulation libre avec un contrôle d'accès à l'auge par des portillons électroniques dans les essais 1, 2 et 3 (Rennes) et en stabulation entravée dans les essais 4 à 8 (Marcenat et Orcival). Les aliments concentrés étaient distribués séparément en 2 ou 3 repas de $30 \mathrm{~min}$ en fonction de la quantité offerte à chaque animal. Le repas de concentré terminé, les animaux recevaient le fourrage à volonté (refus égaux ou supérieurs à $10 \%$ des quantités offertes).

Le concentré de type $\mathrm{A}$, riche en amidon, était constitué principalement de céréales (orge $26 \%$, blé $20 \%$, maïs $20 \%$ ), celui de type $P$, riche en parois très digestibles, de

Tableau 1. Caractéristiques des différents essais. (EM : ensilage de maïs, EH: ensilage d'herbe. $A, P$ et $S$ représentent 3 types d'aliments concentrés : riches en amidon $(A)$, en fibres très digestibles $(P)$ ou moyennement digestibles (S))

\begin{tabular}{|l|c|c|c|c|c|c|c|c|}
\hline $\begin{array}{l}\text { Numéro d'essais } \\
\text { site expérimental }\end{array}$ & $\begin{array}{c}1 \\
\text { Rennes }\end{array}$ & $\begin{array}{c}2^{*} \\
\text { Rennes }\end{array}$ & $\begin{array}{c}3 \\
\text { Rennes }\end{array}$ & $\begin{array}{c}4 \\
\text { Marcenat }\end{array}$ & $\begin{array}{c}5 \\
\text { Marcenat }\end{array}$ & $\begin{array}{c}6 \\
\text { Orcival }\end{array}$ & $\begin{array}{c}7 \\
\text { Orcival }\end{array}$ & $\begin{array}{c}8 \\
\text { Orcival }\end{array}$ \\
\hline $\begin{array}{l}\text { Ration } \\
\begin{array}{l}\text { Fourrage } \\
\text { Concentré }\end{array}\end{array}$ & $\begin{array}{c}\text { EM } \\
\mathrm{A}\end{array}$ & $\begin{array}{c}\mathrm{EM} \\
\mathrm{P}\end{array}$ & $\begin{array}{c}\mathrm{EM} \\
\mathrm{S}\end{array}$ & $\begin{array}{c}\text { Foin } \\
\mathrm{A}\end{array}$ & $\begin{array}{c}\mathrm{EH} \\
\mathrm{A}\end{array}$ & $\begin{array}{c}\mathrm{EH} \\
\mathrm{P}\end{array}$ & $\begin{array}{c}\mathrm{EH} \\
\mathrm{S}\end{array}$ & $\begin{array}{c}\mathrm{EH} \\
\mathrm{S}\end{array}$ \\
\hline $\begin{array}{l}\text { Animaux } \\
\begin{array}{l}\text { Nombre } \\
\text { Primipares }\end{array}\end{array}$ & 9 & $\begin{array}{c}9(6) \\
3(3)\end{array}$ & 9 & 9 & 9 & 9 & 9 & 12 \\
& 3 & 0 & 0 & 3 & 3 & 3 \\
\hline $\begin{array}{l}\text { Semaine de lactation } \\
\text { Moyenne en début d'essai }\end{array}$ & 10 & $8(8)$ & 9 & 10 & 10 & 14 & 13 & 15 \\
\hline $\begin{array}{l}\text { Production de lait en } \\
\text { pré-expérience (kg/j) }\end{array}$ & 32,5 & $34(31,3)$ & 33,3 & 22,8 & 24,3 & 30,2 & 31,1 & 24,5 \\
\hline $\begin{array}{l}\text { Poids vif en } \\
\text { pré-expérience (kg) }\end{array}$ & 560 & $612(592)$ & 615 & 574 & 577 & 594 & 596 & 617 \\
\hline
\end{tabular}

*Les données entre parenthèses indiquent les valeurs des 2 groupes de 3 vaches retenus pour l'analyse statistique 
Tableau 2. Ingestion, production et composition du lait et poids vif (essais 1, 2 et 3, Rennes)

\begin{tabular}{|c|c|c|c|c|c|c|c|c|c|}
\hline & \multicolumn{9}{|c|}{ Ensilage de maïs } \\
\hline & \multicolumn{3}{|c|}{$\begin{array}{c}\text { Essai } 1 \\
\text { Concentré } \mathrm{A}^{(1)} \text { Niveau }\end{array}$} & \multicolumn{3}{|c|}{$\begin{array}{c}\text { Essai } 2 \\
\text { Concentré } \mathrm{P}^{(1)} \text { Niveau }\end{array}$} & \multicolumn{3}{|c|}{$\begin{array}{c}\text { Essai } 3 \\
\text { Concentré } \mathrm{P}^{(1)} \text { Niveau }\end{array}$} \\
\hline & Bas & Moyen & Haut & Bas & Moyen & Haut & Bas & Moyen & Haut \\
\hline Fourrage (kg MS/j) & $13,7^{\mathrm{a}}$ & $12,5^{\mathrm{b}}$ & $11,1^{\mathrm{c}}$ & $15,1^{\mathrm{a}}$ & $13,7^{b}$ & $12,1^{\mathrm{c}}$ & $16,0^{\mathrm{a}}$ & $15,1^{\mathrm{b}}$ & $13,2^{\mathrm{c}}$ \\
\hline Concentré $(\mathrm{kg} \mathrm{MS} / \mathrm{j})^{\{2\}}$ & $4,7^{\mathrm{a}}$ & $6,4^{\mathrm{b}}$ & $8,1^{\mathrm{c}}$ & $3,7^{\mathrm{a}}$ & $5,8^{\mathrm{b}}$ & $7,9^{\mathrm{c}}$ & $4,1^{a}$ & $6,5^{\mathrm{b}}$ & $8,8^{\mathrm{c}}$ \\
\hline Energie ingérée (UFL/j) & $17,0^{\mathrm{a}}$ & $17,9^{\mathrm{b}}$ & $18,6^{c}$ & $16,9^{\mathrm{a}}$ & $17,6^{\mathrm{a}}$ & $18,1^{b}$ & $17,9^{\mathrm{a}}$ & $19,0^{\mathrm{b}}$ & $19,2^{\mathrm{b}}$ \\
\hline Lait brut $(\mathrm{kg} / \mathrm{j})$ & 27,1 & 27,6 & 27,5 & 25,1 & 25,7 & 25,6 & 26,9 & 27,3 & 27,0 \\
\hline Taux butyreux $(\mathrm{g} / \mathrm{kg})$ & 39,9 & 39,6 & 39,8 & 43,2 & 43,6 & 42,8 & 45,1 & 44,0 & 44,7 \\
\hline Taux protéique (g/kg) & $29,9^{\mathrm{a}}$ & $30,5^{\mathrm{a}}$ & $31,3^{b}$ & 31,2 & 31,5 & 31,9 & 32,3 & 32,2 & 32,8 \\
\hline
\end{tabular}

Les moyennes avec des lettres différentes ( $a, b, c)$ sont statistiquement différentes au seuil $5 \%$ ${ }^{(1)}$ Concentré riche en amidon (A), en fibres très digestibles (P) ou moyennement digestibles (S) ${ }^{(2)}$ y compris le concentré expérimental et les minéraux

pulpes de betteraves déshydratées $(55 \%)$ et de coques de soja $(13 \%)$ et celui de type $\mathrm{S}$, riche en parois moyennement digestibles, de son de blé $(55 \%)$ et d'avoine ( $18 \%)$. Le choix des matières premières suivant la nature de l'énergie a conduit à des valeurs énergétiques de ces aliments assez différentes, respectivement $1,14,0,99$ et $0,89 \mathrm{UFL}$ par kg de matière sèche (MS) pour les concentrés $\mathrm{A}, \mathrm{P}$ et $\mathrm{S}$. Les sources protéiques (17 à $24 \%$ de l'aliment concentré) ont été choisies pour correspondre aux mêmes caractéristiques analytiques tout en obtenant un rapport azote sur énergie constant (125 g PDIE/UFL). La valeur nutritive des aliments a été calculée à partir des recommandations en vigueur à l'époque de la mise en place des essais (INRA 1978) car c'est à partir de ces valeurs que les rations correspondant aux traitements ont été établies. Les modifications apportées par les nouvelles recommandations (INRA 1988) ne modifient ni l'interprétation des résultats ni les conclusions de cette étude.

L'ensilage de maïs, récolté dans de bonnes conditions, était d'excellente qualité $(0,89$ UFL/kg MS, $48 \mathrm{~g}$ PDIN et $71 \mathrm{~g}$ PDIE). Le foin provenait d'une prairie naturelle de montagne, déprimée et récoltée dans de bonnes conditions climatiques $(0,65$ UFL, 67 g PDIN et $73 \mathrm{~g}$ PDIE). Les ensilages d'herbe provenaient également de prairies naturelles de montagne récoltées en coupe directe avec une machine double coupe, et additionné d'acide formique $(0,75$ à 0.83 UFL, 89 à $105 \mathrm{~g}$ PDIN et 73 à $81 \mathrm{~g}$ PDIE selon les essais). Les analyses fermentaires (teneurs en ammoniaque et en acide acétique) des différents ensilages utilisés indiquent que leur qualité de conservation était bonne dans les essais 6 et 7 , moyenne dans l'essai 5 et plutôt médiocre dans l'essai 8 (d'après la classification de Dulphy et Demarquilly, 1981).
Les TS pour les 3 aliments concentrés étant comparés en effectuant des apports iso-énergétiques, l'écart d'apport entre 2 niveaux consécutifs variait avec le type d'aliment concentré $(1,75,2,05$ et $2,25 \mathrm{~kg}$ de MS respectivement pour les aliments concentrés de type A, $P$ et $\mathrm{S}$ ). Dans les essais conduits avec des rations à base d'ensilage de maïs, un apport de $200 \mathrm{~g}$ d'urée par jour a été effectué pour rééquilibrer la ration de base en azote fermentescible. De plus, un apport de tourteaux de soja-colza tannés au formaldéhyde a été réalisé pour couvrir les besoins azotés des animaux. Cet apport a été modulé suivant les 3 niveaux $B$, $M$ et $H$ pour que les 3 rations soient iso-azotées. Dans les essais à base de foin et d'ensilage d'herbe, les besoins étant couverts sans apport de tourteaux, seul le concentré expérimental a été utilisé.

\section{Mesures}

Les quantités ingérées ont été mesurées 4 à 5 jours par semaine. Les teneurs en matière sèche des fourrages ont été analysées quotidiennement et celle des aliments concentrés une fois par semaine. La production laitière a été mesurée tous les jours, et les taux butyreux et protéiques 2 jours par semaine. Enfin les animaux ont été pesés une fois par semaine (une fois par quinzaine à Marcenat). Les analyses statistiques ont été effectuées essai par essai suivant un schéma d'analyse de la variance des carrés latins avec 2 (essai 2), 3 (essais 1,3 à 7 ) ou 4 répétitions (essai 8). Dans l'essai 2, un des 3 carrés latins n'a pas été pris en compte dans la mesure où les 3 vaches ont été atteintes de mammites ou ont refusé de consommer les quantités d'aliment concentré prévues par le protocole. Enfin dans l'essai 7, trois données ont été manquantes suite à des problèmes sanitaires 
Tableau 3. Ingestion, production et composition du lait et poids vif (essais 4 et 5 , Marcenat)

\begin{tabular}{|c|c|c|c|c|c|c|}
\hline & \multicolumn{3}{|c|}{ Foin de prairie naturelle } & \multicolumn{3}{|c|}{ Ensilage d'herbe } \\
\hline & \multicolumn{3}{|c|}{$\begin{array}{c}\text { Essai } 4 \\
\text { Concentré } \mathrm{A}^{(1)} \text { Niveau }\end{array}$} & \multicolumn{3}{|c|}{$\begin{array}{c}\text { Essai } 5 \\
\text { Concentré } \mathrm{A}^{(1)} \text { Niveau }\end{array}$} \\
\hline & Bas & Moyen & Haut & Bas & Moyen & Haut \\
\hline Fourrage (kg MS/j) & $12,8^{\mathrm{a}}$ & $12,3^{\mathrm{a}}$ & $11,3^{\mathrm{b}}$ & $12,5^{\mathrm{a}}$ & $11,5^{b}$ & $10,4^{\mathrm{c}}$ \\
\hline Concentré $(\mathrm{kg} \mathrm{MS} / \mathrm{j})^{121}$ & $3,0^{\mathrm{a}}$ & $4,7^{\mathrm{b}}$ & $6,5^{\mathrm{c}}$ & $3,5^{\mathrm{a}}$ & $5,2^{b}$ & $7,0^{c}$ \\
\hline Energie ingérée $(\mathrm{UFL} / \mathrm{j})$ & $11,5^{\mathrm{a}}$ & $13,1^{\mathrm{b}}$ & $14,5^{\mathrm{c}}$ & $13,3^{\mathrm{a}}$ & $14,6^{\mathrm{b}}$ & $15,7^{\mathrm{c}}$ \\
\hline Lait brut $(\mathrm{kg} / \mathrm{j})$ & $15,6^{\mathrm{a}}$ & $16,9^{\mathrm{b}}$ & $18,4^{\mathrm{c}}$ & $18,5^{\mathrm{a}}$ & $18,9^{\mathrm{a}}$ & $20,2^{\mathrm{b}}$ \\
\hline Taux butyreux (g/kg) & 37,8 & 36,9 & 37,2 & 37,6 & 38,1 & 37,5 \\
\hline Taux protéique $(\mathrm{g} / \mathrm{kg})$ & $31,2^{\mathrm{a}}$ & $31,8^{\mathrm{b}}$ & $32,3^{\mathrm{b}}$ & $30,3^{\mathrm{a}}$ & $31,1^{\mathrm{b}}$ & $31,3^{\mathrm{b}}$ \\
\hline
\end{tabular}

Les moyennes avec des lettres différentes $(a, b, c)$ sont statistiquement différentes au seuil $5 \%$

${ }^{(1)}$ Concentré riche en amidon (A)

${ }^{(2)}$ y compris le concentré expérimental et les minéraux

rencontrés par 2 vaches. Ce dernier essai a été recommencé l'année suivante avec 4 répétitions (essai 8).

\section{Résultats}

\section{1 / Quantités ingérées et taux de substitution}

\section{a / Niveaux d'apports de concentrés}

Dans tous les essais, les quantités de fourrages ingérées ont été d'autant plus faibles que la quantité d'aliment concentré était plus importante $(\mathrm{p}<0,01)$ (tableaux 2,3 et 4 ). Le taux de substitution calculé à partir des moyennes de consommation de fourrage et d'aliments concentrés entre les niveaux $\mathrm{B}$ et $\mathrm{M}$ a toujours été plus faible que celui calculé entre les niveaux $M$ et $H$ (tableau 5 et figure 1). Ce phénomène est vérifié quel que soit la nature du fourrage ou de l'aliment concentré. La relation entre le TS et la quantité de MS d'aliment concentré ingérée (MSC) intra essai est hautement significative :

$\mathrm{TS}=$ Essai $+0,093^{*} \mathrm{MSC}$

(ETR $=0,07, \mathrm{R}=0,96, \mathrm{n}=16$ ).

Cependant, près des $2 / 3$ de la somme des carrés des écarts est expliquée par l'effet essai (incluant la nature du fourrage, mais aussi des différences génétiques, climatiques, de pratiques d'élevage...).

\section{b / Nature du fourrage}

Les TS moyens obtenus avec les rations à base d'ensilage de maïs sont toujours beaucoup plus élevés que ceux obtenus avec les rations à base d'ensilage d'herbe $(0,70$ contre
Figure 1. Relation entre le taux de substitution et l'apport moyen d'aliment concentré, ou la valeur énergétique de l'aliment concentré pour 3 fourrages et 3 aliments concentrés.

taux de substitution

0,9

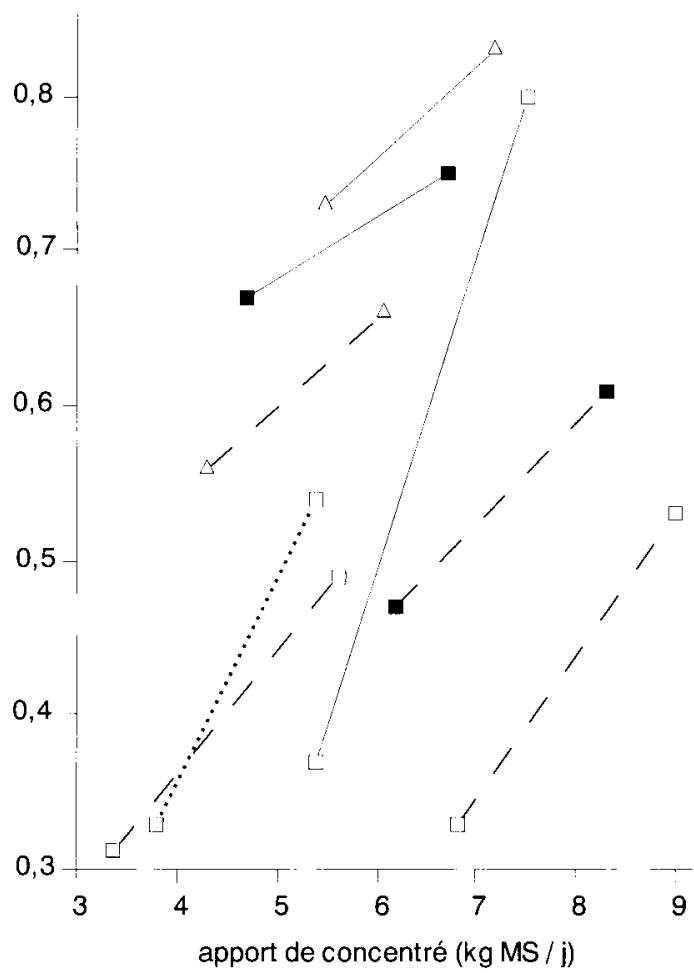

taux de substitution

0,8

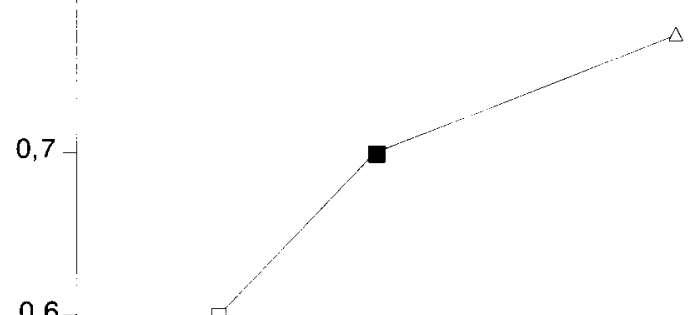

0,6

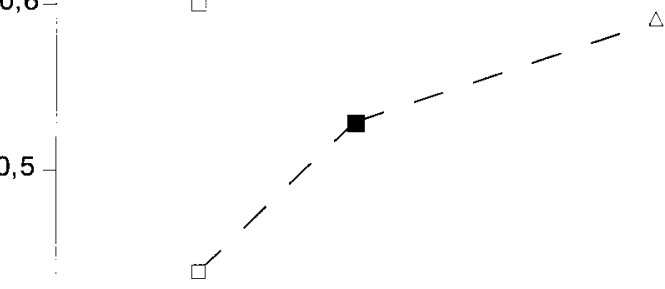

$0,4+$ 0,8 0,9

1,0

1,1

valeur énergétique du concentré (UFL / kg MS)

Fourrage :

Concentré riche en : 
Tableau 4. Ingestion, production et composition du lait et poids vif (essais 6, 7 et 8, Orcival)

\begin{tabular}{|c|c|c|c|c|c|c|c|c|c|}
\hline & \multicolumn{9}{|c|}{ Ensilage d'herbe } \\
\hline & \multicolumn{3}{|c|}{$\begin{array}{c}\text { Essai } 6 \\
\text { Concentré } \mathrm{P}^{(4)} \text { Niveau }\end{array}$} & \multicolumn{3}{|c|}{$\begin{array}{c}\text { Essai } 7 \\
\text { Concentré } \mathrm{S}^{(1)} \text { Niveau }\end{array}$} & \multicolumn{3}{|c|}{$\begin{array}{c}\text { Essai } 8 \\
\text { Concentré } \mathrm{S}^{(1)} \text { Niveau }\end{array}$} \\
\hline & Bas & Moyen & Haut & Bas & Moyen & Haut & Bas & Moyen & Haut \\
\hline Fourrage (kg MS/j) & $12,0^{\mathrm{a}}$ & $11,0^{\mathrm{b}}$ & $9,8^{\mathrm{c}}$ & $12,5^{\mathrm{a}}$ & $11,7^{\mathrm{b}}$ & $10,4^{c}$ & $13,3^{\mathrm{a}}$ & $12,6^{\mathrm{b}}$ & $11,5^{\mathrm{c}}$ \\
\hline Concentrés $(\mathrm{kg} \mathrm{MS} / \mathrm{j})^{|2|}$ & $5,2^{\mathrm{a}}$ & $7,2^{\mathrm{b}}$ & $9,3^{\mathrm{c}}$ & $5,7^{\mathrm{a}}$ & $8,0^{\mathrm{b}}$ & $10,2^{\mathrm{c}}$ & $2,3^{\mathrm{a}}$ & $4,6^{\mathrm{b}}$ & $6,8^{\mathrm{c}}$ \\
\hline Energie ingérée (UFL/j) & $15,1^{\mathrm{a}}$ & $16,3^{\mathrm{b}}$ & $17,2^{\mathrm{c}}$ & $15,5^{\mathrm{a}}$ & $16,8^{\mathrm{b}}$ & $17,7^{\mathrm{c}}$ & $11,9^{\mathrm{a}}$ & $13,4^{b}$ & $14,6^{\mathrm{c}}$ \\
\hline Lait brut $(\mathrm{kg} / \mathrm{j})$ & 25,3 & 25,9 & 26,9 & 25,5 & 26,3 & 26,4 & $19,2^{\mathrm{a}}$ & $21,2^{b}$ & $21,5^{b}$ \\
\hline Taux butyreux $(\mathrm{g} / \mathrm{kg})$ & 37,4 & 36,9 & 36,9 & 35,5 & 37,4 & 36,8 & 34,6 & 35,0 & 35,3 \\
\hline Taux protéique $(\mathrm{g} / \mathrm{kg})$ & 29,0 & 29,8 & 29,9 & 29,3 & 30,2 & 29,5 & $29,0^{\mathrm{a}}$ & $29,7^{\mathrm{b}}$ & $29,9^{b}$ \\
\hline
\end{tabular}

Les moyennes avec des lettres différentes $(\mathrm{a}, \mathrm{b}, \mathrm{c})$ sont statistiquement différentes au seuil $5 \%$

${ }^{11}$ Concentré riche en fibres très digestibles $(\mathrm{P})$ ou moyennement digestibles $(\mathrm{S})$

${ }^{121}$ y compris le concentré expérimental et les minéraux

Tableau 5. Estimation des taux de substitution pour les 3 types de fourrages et d'aliments concentrés

\begin{tabular}{|l|cc|cc|cc|cc|}
\hline \multirow{2}{*}{} & \multicolumn{8}{|c|}{ Nature et niveau des aliments concentrés } \\
\cline { 2 - 10 } & \multicolumn{2}{|c|}{ A } & \multicolumn{2}{|c|}{ P } & \multicolumn{2}{|c|}{ S } & \multicolumn{2}{c|}{ Moyenne } \\
\cline { 2 - 9 } & B-M & M-H & B-M & M-H & B-M & M-H & B-M & M-H \\
\hline Ensilage de maïs & 0,72 & 0,83 & 0,67 & 0,75 & 0,37 & 0,80 & 0,59 & 0,79 \\
Ensilage d'herbe & 0,56 & 0,66 & 0,47 & 0,61 & 0,33 & 0,53 & 0,45 & 0,60 \\
Foin & 0,33 & 0,54 & & & & & & \\
\hline
\end{tabular}

Le taux de substitution est plus élevé avec un concentré riche en amidon qu'avec un concentré riche en fibres, l'écart diminuant lorsque l'apport de concentré augmente.
0,53 en moyenne). Avec le concentré de type A (le seul testé pour les 3 fourrages), les valeurs moyennes de TS calculées sont respectivement de $0,78,0,61$ et 0,44 pour l'ensilage de maïs, l'ensilage d'herbe et le foin. La nature du fourrage entraîne donc d'importantes différences de taux de substitution.

\section{c / Nature du concentré}

Les TS des 3 types d'aliments concentrés ont été comparés avec 2 fourrages différents: ensilage de maïs et ensilage d'herbe. Dans les 2 cas, les TS moyens observés avec le concentré de type $\mathrm{A}(0,70)$ sont supérieurs à ceux obtenus avec celui de type $\mathrm{P}(0,62)$ et plus encore avec celui de type $\mathrm{S}(0,52)$ (tableau 5 ). Cependant ces différences ne sont importantes que dans les comparaisons entre apports de concentrés aux niveaux bas et moyen, en particulier avec le concentré de type $\mathrm{S}$ pour lequel le TS est très faible. Avec ce type de concentré, peu encombrant en faible quantité, les phénomènes de substitution augmentent beaucoup plus rapidement avec le niveau d'apport qu'avec les 2 autres types de concentrés étudiés.

\section{2 / Production et composition du lait}

La réponse de production de lait observée entre les lots $B$ et $H$ dépend de la nature du fourrage utilisé (tableaux 2,3 et 4 ). Avec l'ensilage de maïs, aucune réponse n'a été obtenue (respectivement $+0,4,+0,3,-0,2 \mathrm{~kg}$ de lait $4 \%$ pour les essais 1,2 et 3 ). Avec l'ensilage $\mathrm{d}$ 'herbe les réponses ont été plus importantes et significatives $(\mathrm{P}<0,05)$ dans 3 des 4 essais (respectivement $+1,6,+1,3,+1,3$ et $+2,3 \mathrm{~kg}$ de lait $4 \%$ pour les essais $5,6,7$ et 8). Enfin, la réponse maximale a été observée avec le régime à base de foin, $(+2,5 \mathrm{~kg}$ de lait $4 \%$, essai $4, P<0,01$ ), avec lequel le niveau de production était le plus faible.

Aucun essai n'a permis de mettre en évidence un effet significatif du niveau d'apport d'aliments concentrés sur le taux butyreux. Par contre, dans tous les essais, l'augmentation des apports énergétiques s'est accompagnée d'un accroissement du taux de protéines compris entre 0,2 et $1,4 \mathrm{~g} / \mathrm{kg}$ de lait entre les 
lots $B$ et $H$, cette augmentation étant significative dans 4 des 8 essais $(P<0,01$ ) (tableaux $2,3$ et 4$)$.

\section{Discussion}

\section{1 / Niveau d'apport d'aliments concentrés}

Cette série d'essais confirme que quelle que soit la nature du fourrage considéré, le TS s'accroît avec la quantité d'aliments concentrés dans la ration. Ce résultat observé précédemment par plusieurs auteurs sur vaches laitières (Lamb et al 1973, Vérité et Journet, 1973, Ostergaard 1979, Laird et al 1981) confirme ceux obtenus sur bovins en croissance ou à l'engrais (Berge et Dulphy 1985). Le cœfficient d'augmentation du TS de 0,093 point/kg MS de concentré ingéré (équation 1) est très proche de celui obtenu par Ostergaard (1979). Cet accroissement n'est cependant pas observé de façon systématique (cf revue de Thomas 1987), vraisemblablement en raison des différences de durées expérimentales servant à calculer les taux de substitution. En effet, à long terme, l'augmentation du niveau d'apport de concentré s'accompagne d'une augmentation de la production laitière et donc de la capacité d'ingestion ce qui atténue les diminutions de consommation de fourrage. Ainsi, lorsque les quantités ingérées sont corrigées des différences de niveau de production, les TS augmentent avec la quantité d'aliments concentrés ingérée alors qu'ils restent constants sans cette correction (Kristensen et Ingvartsen 1985, cités dans Thomas 1987). A court terme, les réponses plus faibles observées sur la production laitière dues à la mobilisation des réserves permettent de penser que la capacité d'ingestion n'est que peu modifiée.

\section{2 / Nature du fourrage}

Contrairement à l'objectif recherché dans le protocole, il est clair que le niveau moyen d'apport d'aliments concentrés ne correspond vraisemblablement pas à la même situation énergétique d'un fourrage à l'autre. Les différences de réponse de la production de lait à un supplément d'aliment concentré observées entre les 3 fourrages étudiés le démontrent. Ce biais provient en partie d'une erreur d'appréciation de la valeur énergétique des différents fourrages utilisés au moment de l'élaboration du protocole, mais surtout du fait qu'avec un ensilage de maïs de bonne qualité, l'apport de concentré nécessaire à la couverture des besoins azotés conduit à réaliser une ration dont la couverture énergétique est déjà supérieure à $25 \mathrm{~kg}$ de lait. Pour cette raison, les TS observés avec des ensilages de maïs de bonne qualité sont généralement élevés (Vérité 1972, Vérité et Journet 1973, Vérité et al 1980). Pour obtenir une situation énergétique comparable à celle du foin ou de l'ensilage d'herbe, il aurait fallu disposer d'animaux ayant un potentiel de production bien supérieur à ceux utilisés.
Figure 2. Relation entre le taux de substitution divisé par la valeur énergétique de l'aliment concentré (TS/UFLc) et la réponse de la production laitière $4 \%$ observée avec le supplément d'aliment concentré.

TS / UFLc

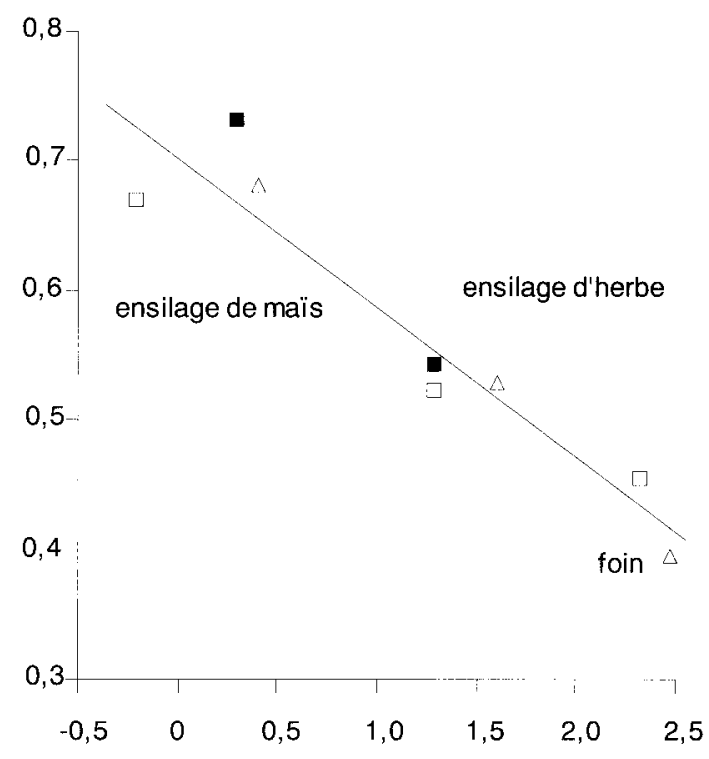

réponse de la production laitière $4 \%(\mathrm{~kg} / \mathrm{j})$

Concentré riche en : $\triangle$ amidon

- fibres très digestibles

$\square$ fibres moyennement digestibles

La comparaison entre le foin et l'ensilage d'herbe (essais 4 et 5) réalisée à Marcenat avec le concentré de type $\mathrm{A}$ montre qu'avec des ingestibilités comparables entre le foin et l'ensilage d'herbe, les phénomènes de substitution sont plus importants avec l'ensilage d'herbe dont la valeur énergétique est supérieure à celle du foin. La nature du fourrage semble donc intervenir dans les phénomènes de substitution non seulement par l'ingestibilité du fourrage, comme cela avait été proposé dans la première version du système des unités d'encombrement (INRA 1979, Jarrige et al 1986), mais aussi par sa valeur énergétique et le bilan énergétique qu'il engendre.

\section{3 / Nature de l'aliment concentré}

Les résultats obtenus indiquent que les phénomènes de substitution sont modifiés par la nature de l'aliment concentré, au moins aux niveaux auxquels ces aliments ont été distribués. L'effet de la nature du concentré sur le taux de substitution chez la vache laitière a déjà été étudié par plusieurs auteurs qui ont obtenu soit un taux légèrement supérieur avec le concentré de type A par rapport au type $\mathrm{P}$ (Dulphy et al 1987), soit des taux identiques (Thomas et al 1986, Sutton et al 1987). Les études réalisées sur moutons (Berge et Dulphy 1985) avaient conclu que les concentrés de type $\mathrm{P}$ étaient plus encombrants à 
faible niveau d'apport que ceux de type A mais que la tendance s'inversait rapidement lorsque la proportion d'aliments concentrés dépassait $50 \%$ de la ration.

Aux niveaux moyens auxquels ces aliments concentrés ont été distribués, il semble bien que les concentrés de type $P$ soient légèrement moins encombrants et présentent des phénomènes de substitution un peu moins importants (à condition qu'ils aient une valeur énergétique plus faible que celle des concentrés de type A, ce qui est généralement le cas), ce que confirme dans l'ensemble les données de la littérature (Revue de Coulon et al 1989). D'un point de vue zootechnique, la différence reste cependant peu importante.

Le concentré de type $\mathrm{S}$ a été peu étudié jusqu'à présent, mais Vérité et Dulphy (1981) ont dèjà mis en évidence des TS plus faibles avec le type $\mathrm{S}$ qu'avec le type $\mathrm{A}$. Cependant, lorsque le concentré représente $60 \%$ de la ration, l'ingestion est considérablement réduite $(-27 \%$ et $-15 \%$ respectivement chez des moutons et des vaches) avec un concentré de type S par rapport à un concentré de type $\mathrm{P}$ (Doreau et al 1988). Le concentré de type S semble donc assez particulier dans la mesure où son encombrement, très faible lorsqu'il est distribué en faible quantité, s'accroît plus rapidement quand sa proportion dans la ration augmente que celui d'un concentré de type $P$. Ce résultat avait déjà été obtenu sur moutons (Berge et Dulphy 1985). Le nombre d'essais sur ce type d'aliments ne permet toutefois pas de conclure avec certitude sur cet effet et sur les mécanismes qui pourraient l'expliquer.

Il semble donc que l'effet de la nature de l'aliment concentré sur les phénomènes de substitution ne puisse être totalement ignoré. La figure 1 montre qu'en moyenne, aux niveaux auxquels ces aliments concentrés ont été utilisés, les TS moyens des essais réalisés avec des ensilages d'herbe et de maïs augmentent avec la valeur énergétique de l'aliment concentré. Ceci ne permet cependant pas d'expliquer en totalité les évolutions rapide du TS avec le concentré de type $\mathrm{S}$.

\section{4 / Substitution et bilan énergétique}

En fait les 3 facteurs de variation des TS étudiés dans ces essais (proportion d'aliment concentré, nature du fourrage et de l'aliment concentré) semblent pouvoir être réunis dans un seul et même concept physiologique : le niveau de satisfaction des besoins énergétiques de l'animal. Cette notion semble bien démontrée par ces essais pour l'effet du niveau d'apport d'aliment concentré. Elle peut être étendue à la nature des composants de la ration. En effet, les résultats obtenus dans les 8 essais montrent l'existence d'une relation entre les valeurs de TS et les réponses de production laitière $4 \%$ obtenues (dPL4 \%) entre les niveaux $\mathrm{B}$ et $\mathrm{H}$ d'aliments concentrés :
$\mathrm{TS}=0,7 \cdot 0,11^{*} \mathrm{dPL} 4 \%$

$(\mathrm{ETR}=0,09, \mathrm{R}=-0,77, \mathrm{n}=8)$

Pour étendre cette notion d'énergie à la nature de l'aliment concentré, il est possible de rapporter les TS obtenus à la valeur énergétique des aliments concentrés utilisés (UFLc). L'équation de prédiction du rapport TS/UFLc en fonction de la réponse de production de lait est identique à celle obtenue avec TS, ce qui est logique car la valeur énergétique moyenne des 3 types de concentrés est de $1 \mathrm{UFL} / \mathrm{kg}$ de MS, mais la corrélation est beaucoup plus étroite ( $\mathrm{ETR}=0,04, \mathrm{R}=-0,96$ ) (figure 2). Si les valeurs de ces cœfficients ne sont qu'indicatives, car les différents essais ont été réalisés avec des animaux ayant des niveaux de production très différents, la dernière relation conforte l'hypothèse d'un lien étroit entre les phénomènes de substitution et le bilan énergétique de l'animal (figure 3). Les phénomènes de substitution en pleine lactation seraient donc directement fonction de la situation énergétique de l'animal lorsque celui-ci reçoit un supplément d'aliment concentré, comme l'avaient déjà proposé Vérité et al (1980). Les résultats obtenus par cette série d'essais nécessiteraient cependant d'être confortés notamment avec des apports plus élevés d'aliments concentrés pour des fourrages de plus faible valeur énergétique.

Figure 3. Relation entre le taux de substitution et le bilan énergétique à faible niveau d'apport de concentré, pour 3 types de concentrés et 3 fourrages $(M$ : ensilage de mails, $H$ : ensilage $d$ herbe, $F$ : foin).

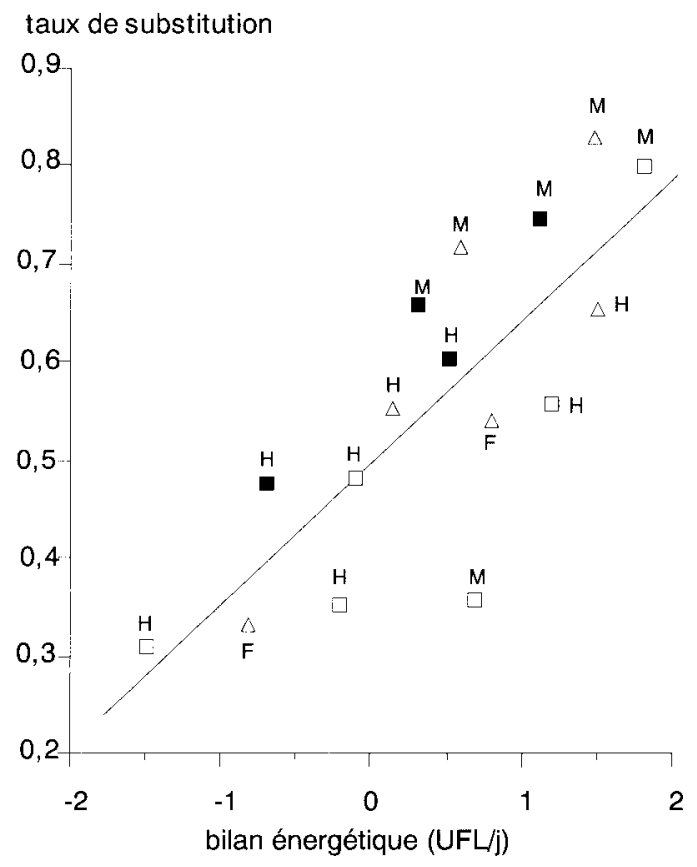

Concentré riche en : $\Delta$ amidon

- fibres très digestibles

$\square$ fibres moyennement digestibles
Chez les vaches en milieu de lactation, la substitution fourrage-concentré est d'autant plus importante que le bilan énergétique de l'animal augmente. 
Tous les facteurs susceptibles de modifier cette situation énergétique, aussi bien par les apports de la ration que par les besoins de l'animal, pourraient influer sur le TS. La prédiction des phénomènes de substitution devra donc se faire préférentiellement à partir de l'équilibre énergétique de la ration. C'est à partir de ce principe qu'ont été proposées, dans le cadre du système des U.E., de nouvelles méthodes d'estimation de la valeur d'encombrement ou de rassasiement de l'aliment concentré pour les vaches laitières (Duplhy et al 1987, Faverdin et al 1987)

\section{Références bibliographiques}

Berge, P. Dulphy, J.P., 1985. Etude des interactions entre fourrage et aliment concentré chez le mouton. 1. Facteurs de variation du taux de substitution. Ann. Zootech., 34: 313-334.

Bertilsson, J., Burstedt, E., 1983. Effects of conservation method and stage of maturity upon the feeding value of forages to dairy cows. Swedish J. Agric. Res., 13: 189-200.

Butler, T.M., Gleeson, P.A., Morgan, D.J., 1983. Effect of supplement feeding level and crude protein content of the supplement on the performance of spring-calving dairy cows. Ir. J. Agric. Res., 22: 69-78.

Castle, M.E., Gill, M.S., Watson, J.N., 1981. Silage and milk production : a comparison between barley and dried sugar-beet pulp as silage supplements. Grass Forage Sci., 36: 319-324.

Coulon, J.B., Faverdin, P..Laurent, F., Cotto, G., 1989. Influence de la nature de l'aliment concentré sur les performances des vaches laitières. INRA Prod. Anim., 2(1): 47-53.

Doreau, M., Michalet-Doreau, Brigitte, Poncet, C., Le Guen, Marie-Pierre, 1988. Comparative rumen digestion of bran and beet pulp in sheep and dairy cows. Reprod. Nutr. Dévelop., 28: 119-120.

Dulphy, J.P., 1978. Quantités ingérées et phénomènes de substitution; Conséquences pour le rationnement. In: La vache laitière, INRA Publications (Ed.), Route de St-Cyr, 78000, Versailles, 87-98.

Dulphy, J.P., Andrieu, J.P., Rouel, J., 1987. Effet de la nature de l'aliment concentré sur les performances de vaches laitières recevant une ration à base de foin. Bull. Tech. CRZV Theix, INRA, 67; 43-47.

Dulphy, J.P., Demarquilly C., 1981. Problèmes particuliers aux ensilages : in "Prévision de la valeur nutritive des aliments des Ruminants". Ed. INRA Publications, Route de St-Cyr, Versailles, p. 86.

Faverdin, P., Dulphy, J.P., Coulon, J.B., Vérité, R., Garel, J.P., Rouel, J., Marquis, B., 1991. Substitution of roughage by contrate for dairy cows. Livest. Prod. Sci., 27: 137-156.

Faverdin, P., Hoden, A., Coulon, J.B., 1987. Recommandations alimentaires pour les vaches laitières. Bull. Tech. C.R.Z.V. Theix, I.N.R.A., 70: 133-152

Gordon, F.J., 1984. The effect of level of concentrate supplementation given with grass silage during the winter on the total lactation performance of autumn-calving dairy cows. J. Agric. Sci. Camb., 102: 163-179.

INRA, 1979. Le système des unités d'encombrement pour les bovins. Bull. Techn. C.R.Z.V. Theix, 38: 57-79.

Jarrige, R., Demarquilly, C., Dulphy, J.P., Hoden, A., Robelin, J., Geay, Y., Journet, M., Malterre, C., Micol, D., Petit, M., 1986. The INRA "Fill unit" system for predicting the voluntary intake of forage-based diets in ruminants : a review. J. Anim. Sci., 63: 1737-1758.

Kristensen, V.F., 1983. Styring af foderoptagelsen ved hjaelp af foderrationens sammensaetning og valg af fodringsprincip. In: Optimale foderrationer til malkekœn. Fodervaerdi, foderoptagelse, omsaetning og pro- duktion. Ostergaard, V. and Neimann-Sorensen, A. (Eds.). Beretning fra Statens Husdyrbrugsforsorg, 551 7.1-7.35.

Laird, R., Leaver, J.D., Moisey, F.R., Castle, M.E., 1981. The effects of concentrate supplements on the performances of dairy cows offered grass silage ad libitum. Anim. Prod., 33: 199-209.

Lamb, R.C., Stoddard, G.E., Mickelsen, C.H., Anderson, M.J., Waldo, D.R., 1973. Response to concentrates containing two percents of protein fed at four rates for complete lactations. J. Dairy Sci., 57: 811-815.

Montgomery, M.J., Culvahouse, E.W., Henderson, H.A., 1971. Effect of concentrate supplementation on the voluntary intake of high and low quality forages. J. Dairy Sci., 54: 772(Abstr.P23).

Ostergaard, V., 1979. Strategies for concentrate feeding to attain optimum feeding level in high yielding dairy cows. Chap. V - Food intake. Beretning fra statens husdyrbrugs forsog, 482: 37-67.

Steen, R.W., Gordon, F.J., 1980a. The effect of level and system of concentrate allocation to January/February calving cows on total lactation performance. Anim. Prod., 30: $39-51$.

Steen, R.W., Gordon, F.J., 1980b. The effect of type of silage and level of concentrate supplementation offered during the early lactation on total lactation performance of January/February calving cows. Anim. Prod., 30: 341-354.

Sutton, J.D., Bines, J.A., Morant, S.V., Napper, D.J., 1987. A comparison of starchy and fibrous concentrates for milk production, energy utilisation and hay intake by Friesian cows. J. Agric. Sci. Camb., 109: 375-386.

Thomas, C., 1987. Factors affecting substitution rates in dairy cows on silage based rations. In: Recent advence in animal nutrition - 1987. Studies in agricultural and food sciences. Butterworths, London, 233 p, 205-218.

Thomas, C. Aston, K., Daley, S.R., Bass, J., 1986. Milk production from silage. 4 . The effect of the composition of the supplement. Anim. Prod. 42: 315-325.

Vérité, R., 1972. Complémentation des rations à base d'ensilage de maïs en début de lactation. Bull. Techn. C.R.Z.V. Theix, 7: 5-9.

Vérité, R., Dulphy, J.P., 1981. Effet de la nature de l'aliment concentré sur l'ingestion et les performances des vaches laitières. Bull. Techn. C.R.Z.V. Theix, INRA, 45: 15-21.

Vérité, R., Journet, M., 1973. Complémentation des rations à base d'ensilage de maiis en début de lactation. Bull. Techn. C.R.Z.V. Theix, 11:41-46.

Vérité, R., Journet, M., Rémond, B., 1980. Energy supplementation of maize silage rations for dairy cows. in "Production and utilization of the maize crop". Proc. of the 1st european maize congress. Euro Mais 1979. E.S. Bunting (Ed.), 307-315.

Vinet, C., Bouchard, R., St-Laurent, G.J., 1980. Effects of stage of maturity of timothy hay and concentrate supplementation on performance of lactating dairy cows. Can J. Anim. Sci., 60: 511-521. 


\section{Summary}

Substitution of roughage by concentrates in dairy cows.

The effects of the concentrate supplementation level, the type of roughage and the type of concentrate on the substitution rate (SR) between roughage and concentrates have been studied over a course of 8 trials in three experimental sites. In each trial, the 3 levels of concentrates were compared using a $3 \times 3$ Latin square design, repeated with 3 or 4 groups of Holstein-Friesian crossbred cows in mid-lactation, each period lasting 1 month. The 8 trials corresponded to 8 different diets according to the type of roughage and the type of concentrates used. The SRs increase systematically with the concentrate supplementation level, on average 0.47 between $L$ and $M$ and 0.67 between $M$ and $H$. For a given roughage, the increase in the energy concentration of the concentrate corresponds to an increase in the SR values, mainly between $L$ and $M$. The average SR values observed when using maize silage $(0.7)$ are higher than those observed with grass silage (0.53) and also for the hay (0.44). These trials show, looking at the 3 variation factors in the study, that in mid-lactation, the higher the energy balance is, the more important the SR values become.

FAVERDIN P., DULPHY J.P., COULON J.B., VERITE R., GAREL J.P., ROUEL J., MARQUIS B. Les phénomènes de substitution fourrages-concentrés chez la vache laitère. INRA Prod. Anim., 5 (2), 127 - 135. 